\title{
Preface to the Special Issue of the International Symposium on Single-Atom Catalysis (ISSAC-2016)
}

Catalysis is one of the most important chemical processes for production of goods and for environmental remediation. Almost $80 \%$ of chemical manufacturing involves either heterogeneous or homogeneous catalysts, mostly consisting of expensive noble metals. Designing highly active, selective and stable catalysts with most efficient use of rare and expensive metals becomes Grand Challenge of heterogeneous catalysis and is critical to the sustainable development of our planet.

Sir Hugh Stott Taylor in 1925 proposed the concept of active centers on solid surfaces. The active centers of homogeneous catalysts are well-defined and understood. The scenario is much more complicated for heterogeneous catalysts, where the active centers for a specific catalytic reaction is often unclear or unknown. Since a variety of active centers often exist on heterogeneous surfaces or are created during catalytic reactions the selectivity control of a given reaction is limited because undesirable reactions can occur on the various types of active centers. The search to have a catalyst that possesses highest atom-utilization efficiency and well-defined, stable active centers was recently realized with the direct synthesis of supported single metal atoms as catalysts. With extensive characterizations, catalytic evaluations, and density functional theory calculations it was unambiguously confirmed that isolated single metal atoms dispersed onto high-surface-area supports not only mediate catalysis but also can be extremely stable and selective. Therefore, the concept of single-atom catalysis (SAC) was proposed and many recent examples firmly corroborated this proposal. The catalysis community quickly accepted the SAC concept and as a result the original paper on SAC was listed as one of the 10 highly cited papers among the 100 issues of Nature Chemistry.

To expedite the research progress on SAC, we organized The First International Symposium on Single-Atom Catalysis (ISSAC-2016) at Dalian Institute of Chemical Physics in Dalian, China, with invited presentations and contributed posters. As a follow up of the ISSAC-2016, this special issue collected 23 papers with 1 viewpoint, 6 perspectives, 2 full reviews, 2 minireviews and 12 original research papers. It is expected that this special collection of papers by active and pioneering researchers in this field will benefit the broader catalysis community. As a series of symposia on SAC, the Second International Symposium on Single-Atom Catalysis (ISSAC-2018) will be held at Tsinghua University in Beijing, China June 2018. We hope that the series of special symposia on SAC will promote the development of SAC in both fundamental research and practical applications.

We sincerely appreciate the time and efforts of all the authors and reviewers for their significant contributions to this special issue. We are indebted to the editorial staff for their hardworking in editing and publishing this special issue.

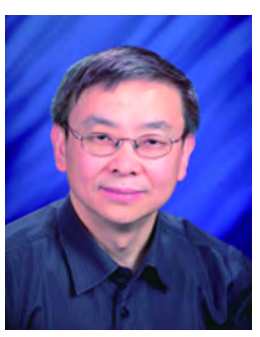

Prof. Jun Li

Department of Chemistry and Key Laboratory of Organic Optoelectronics \& Molecular Engineering of Ministry of Education, Tsinghua University, China E-mail: junli@mail.tsinghua.edu.cn

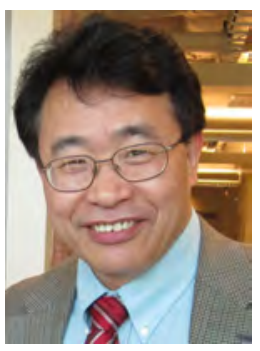

Prof. Jimmy (Jingyue) Liu Department of Physics, Arizona State University, United States E-mail: Jingyue.Liu@asu.edu

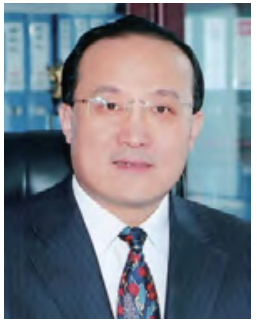

\section{Prof. Tao Zhang}

Dalian Institute of Chemical Physics Chinese Academy of Sciences, China E-mail: taozhang@dicp.ac.cn 